\title{
Article
}

\section{The Kinase Chemogenomic Set (KCGS): An Open Science Resource for Kinase Vulnerability Identification}

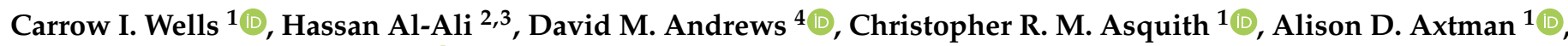 \\ Ivan Dikic ${ }^{5,6,7}$, Daniel Ebner ${ }^{8}$ (D), Peter Ettmayer ${ }^{9}$, Christian Fischer ${ }^{10}$, Mathias Frederiksen ${ }^{11}$, Robert E. Futrell ${ }^{1}$, \\ Nathanael S. Gray ${ }^{12,13}$, Stephanie B. Hatch ${ }^{14}$, Stefan Knapp ${ }^{6,15,16}{ }^{\circledR}$, Ulrich Lücking ${ }^{17}$, Michael Michaelides ${ }^{18}$, \\ Caitlin E. Mills ${ }^{19}$, Susanne Müller 6,15,16®D, Dafydd Owen ${ }^{20}$, Alfredo Picado ${ }^{1}$, Kumar S. Saikatendu ${ }^{21}$, \\ Martin Schröder 6,15,16, Alexandra Stolz 5,6,15(D, Mariana Tellechea 5,6,15, Brandon J. Turunen 22, Santiago Vilar ${ }^{3}$, \\ Jinhua Wang ${ }^{12,13}$, William J. Zuercher ${ }^{1}{ }^{\mathbb{D}}$, Timothy M. Willson ${ }^{1}$ and David H. Drewry ${ }^{1, *(1)}$
}

check for

updates

Citation: Wells, C.I.; Al-Ali, H.; Andrews, D.M.; Asquith, C.R.M.; Axtman, A.D.; Dikic, I.; Ebner, D.; Ettmayer, P.; Fischer, C.; Frederiksen, M.; et al. The Kinase Chemogenomic Set (KCGS): An Open Science Resource for Kinase Vulnerability Identification. Int. J. Mol. Sci. 2021, 22 566. https://doi.org/10.3390/ ijms22020566

Received: 8 December 2020 Accepted: 29 December 2020 Published: 8 January 2021

Publisher's Note: MDPI stays neutral with regard to jurisdictional clai$\mathrm{ms}$ in published maps and institutional affiliations.

Copyright: (C) 2021 by the authors. Licensee MDPI, Basel, Switzerland. This article is an open access article distributed under the terms and conditions of the Creative Commons Attribution (CC BY) license (https:// creativecommons.org/licenses/by/ $4.0 /)$.
1 Structural Genomics Consortium, UNC Eshelman School of Pharmacy, University of North Carolina at Chapel Hill, Chapel Hill, NC 27599, USA

2 The Miami Project to Cure Paralysis, Peggy and Harold Katz Family Drug Discovery Center, Sylvester Comprehensive Cancer Center, Departments of Neurological Surgery and Medicine, University of Miami Miller School of Medicine, Miami, FL 33136, USA

3 Truvitech LLC, Miami, FL 33136, USA

4 AstraZeneca, Darwin Building, Cambridge Science Park, Cambridge CB4 0WG, UK

5 Institute of Biochemistry 2, Faculty of Medicine, Goethe University Frankfurt, Theodor-Stern-Kai 7, 60438 Frankfurt am Main, Germany

6 Buchmann Institute for Molecular Life Sciences, Goethe University Frankfurt, Max-von-Laue-Str. 15, 60438 Frankfurt am Main, Germany

7 Max Planck Institute of Biophysics, Max-von-Laue-Str. 3, 60438 Frankfurt am Main, Germany

8 Nuffield Department of Medicine, Target Discovery Institute, University of Oxford, Oxford OX3 7FZ, UK

9 Boehringer Ingelheim RCV GmbH \& Co KG, 1121 Vienna, Austria

10 MSD, 33 Avenue Louis Pasteur, Boston, MA 02115, USA

11 Novartis Institutes for BioMedical Research, Novartis Campus, 4056 Basel, Switzerland

12 Department of Biological Chemistry and Molecular Pharmacology, Harvard Medical School, Boston, MA 02115, USA

13 Department of Cancer Biology, Dana-Farber Cancer Institute, Boston, MA 02215, USA

14 MRC Oxford Institute for Radiation Oncology, Department of Oncology, University of Oxford, Roosevelt Drive, Oxford OX3 7XB, UK

15 Structural Genomics Consortium, Buchmann Institute for Molecular Life Sciences, Goethe University Frankfurt, Max-von-Laue-Straße 15, 60438 Frankfurt am Main, Germany

16 Institute for Pharmaceutical Chemistry, Johann Wolfgang Goethe-University, Max-von-Laue-Str. 9, 60438 Frankfurt am Main, Germany

17 Bayer Pharma AG, Drug Discovery, Müllerstrasse 178, 13353 Berlin, Germany

18 Oncology Discovery, AbbVie, 1 North Waukegan Road, North Chicago, IL 60064, USA

19 Laboratory of Systems Pharmacology, Department of Systems Biology, Harvard Medical School, Boston, MA 02115, USA

20 Discovery Network Group, Pfizer Medicine Design, Cambridge, MA 02139, USA

21 Global Research Externalization, Takeda California, Inc., 9625 Towne Center Drive, San Diego, CA 92121, USA

22 GlaxoSmithKline, Chemical Biology, 1250 S Collegeville Rd, Collegeville, PA 19426, USA

* Correspondence: David.Drewry@unc.edu

Abstract: We describe the assembly and annotation of a chemogenomic set of protein kinase inhibitors as an open science resource for studying kinase biology. The set only includes inhibitors that show potent kinase inhibition and a narrow spectrum of activity when screened across a large panel of kinase biochemical assays. Currently, the set contains 187 inhibitors that cover 215 human kinases. The kinase chemogenomic set (KCGS), current Version 1.0, is the most highly annotated set of selective kinase inhibitors available to researchers for use in cell-based screens.

Keywords: protein kinase; kinase inhibitor; chemogenomic set; phenotypic screening; small molecules; KCGS; drug discovery; druggable genome; understudied kinase 


\section{Introduction}

The protein kinases have emerged as one of the most productive families of drug targets in the 21st century. Over 60 small molecule kinase inhibitors have been approved by the FDA since 2001 for the treatment of cancer, inflammation, and fibrosis [1]. Many of these drugs, specifically those that are used in oncology, owe their efficacy to inhibition of multiple kinases [2]. For some targets second- and third-generation inhibitors have been designed to block the activity of mutant kinases that cause drug resistance after first line therapy. Collectively these drugs target only a small fraction of the 500+ human kinases. We and others have proposed that the remaining kinases represent an untapped trove of new drug targets [3-5].

Despite the concerted efforts of academic and industrial scientists over the past 25 years, the vast majority of the human kinases remain understudied. Various bibliographic analyses show that, similar to many other protein families, $90 \%$ of the research effort has been expended on $<20 \%$ of the kinases [6]. Initiatives such as the $\mathrm{NIH}$-sponsored Illuminating the Druggable Genome (IDG) program have sought to change this dynamic by making available high-quality data sets and research tools for the "dark" kinases [7]. The availability of a set of potent and selective inhibitors of the understudied kinases could greatly aid the study of their biology and uncover new targets for drug development.

An ever-growing number of kinase inhibitors are commercially available. Many of these compounds have advanced to clinical studies and may be useful for investigators seeking to repurpose kinase drugs for a secondary indication but they do little to expand the number of new kinase targets [8]. Notably, the commercially available kinase inhibitors vary widely in the amount and depth of annotation provided. Their vendors typically list the primary target of each inhibitor and perhaps a handful of off-targets but only rarely provide kinase selectivity profiles. Although the amount of kinase profiling data that can be found in public databases is growing [9], for many compounds broad profiling is either unavailable or described in a multitude of assay formats in the supporting information that accompanies a primary publication. While commercially available kinase sets contain valuable inhibitors of the well-studied kinases, they do little to provide tools to expand research across the breadth of the kinome.

The public availability of a high-quality chemical probe for every understudied kinase would be an ideal way to embolden researchers to explore the therapeutic potential of each kinase target [10]. However, the development of potent and selective chemical probes for over 500 kinases would be an insurmountable task using current resources and technologies. A chemogenomic set [11] of kinase inhibitors provides a practical solution to the problem [12]. The majority of kinase inhibitors, by virtue of competing with the common cofactor adenosine triphosphate (ATP) in a highly conserved binding site, invariably show some cross-activity on multiple kinases. Landmark studies by BristolMyers Squibb and GlaxoSmithKline scientists showed that kinome-wide profiling could identify inhibitors with collateral activity on the understudied kinases [13,14]. Building on these observations, the kinase inhibitor sets PKIS [15] and PKIS2 [16] were assembled as collections of published kinase inhibitors using the principles that chemical diversity and the inclusion of multiple exemplars of each chemotype would increase the breadth of kinase coverage and aid analysis of phenotypic screening data [17]. Both sets have found widespread use in the research community and demonstrated that repurposed inhibitors from past projects could be used to probe the biology of the kinase they were made to target and their off-targets as well. However, the full kinase profile of each inhibitor in PKIS and PKIS2 was not known in advance of their selection, and as a result both sets contained many inhibitors that were either too promiscuous (inhibition of too many kinases) or lacked sufficient target potency to be useful contributors to a chemogenomic set [18]. In spite of this limitation, PKIS and PKIS2 contained many valuable inhibitors for a broad set of understudied kinases. Encouraged by these results, we proposed a community experiment to build an optimized kinase chemogenomic set (KCGS) to cover every human kinase [16]. 
Each inhibitor would have its full kinome profile determined in advance and only those compounds that met a prespecified potency and selectivity would be added to KCGS.

From the start, we chose to make KCGS an open science experiment. All of the compound structures and associated kinase inhibition and selectivity data would be made publicly available. KCGS would be distributed under a Material Trust Agreement (Supplementary File S1) that supported its use as a public resource and prevented the recipients from blocking other researchers from using the set [19]. Eight pharmaceutical companies answered the call to donate kinase inhibitors from their internal compound collections to the effort. Many but not all of these companies were current partners in the Structural Genomics Consortium (SGC) [20]. In addition, several leading academic groups contributed compounds to the initiative. To date, over 1200 kinase inhibitors have been profiled as candidates for inclusion into KCGS. Here, we present the first version of KCGS as well as its initial characterization and examples of its use in cell-based assays. The set will be broadly useful to the scientific community for phenotypic screening to identify the roles of various kinases in biology and disease.

\section{Results}

\subsection{Compound Selection}

Candidate kinase inhibitors were received from GlaxoSmithKline, Pfizer, Takeda, Abbvie, MSD, Bayer, Boehringer Ingelheim, and AstraZeneca. In addition, Vertex gave permission to include their commercially available inhibitors. Academic laboratories that donated inhibitors were Cancer Research UK, Nathanael Gray, and multiple SGC sites. In total, 250 new inhibitors were donated to the initiative as candidates to complement the 950 inhibitors of PKIS and PKIS2.

At the outset, we selected the DiscoverX scanMAX assay to profile all kinase inhibitors donated to the initiative [21]. The scanMAX assay provided kinase binding data on 401 wild type human kinases (Table 1), which was at the time the broadest coverage by any single assay panel [22]. All kinase inhibitors were profiled at a concentration of $1 \mu \mathrm{M}$. Using a cut-off of $10 \%$ activity remaining (PoC, equivalent to $90 \%$ inhibition), an activity profile was determined for each inhibitor and a selectivity index $\left(\mathrm{S}_{10}\right)$ was calculated as the fraction of kinases meeting the cut-off. Compounds with an $S_{10}(1 \mu \mathrm{M})<0.04$ were initially selected for consideration for inclusion in KCGS. For these compounds, we performed full-dose response experiments in order to determine $\mathrm{K}_{\mathrm{D}}$ values for all kinases with $\mathrm{PoC}<10 \%$ in the scanMAX experiment.

Table 1. Kinase coverage by kinase chemogenomic set (KCGS) version 1 . The human kinases are divided into 10 subfamilies [23]. Kinases: the number of human kinases in each subfamily. Assays: the number of kinases in the DiscoverX scanMAX panel. KCGS: The number of kinases covered by an inhibitor. \%: The percentage of kinases screened that are covered by an inhibitor.

\begin{tabular}{ccccc}
\hline & Kinases & Assays & KCGS & \% \\
\hline AGC & 63 & 46 & 20 & 43 \\
Atypical & 34 & 7 & 5 & 71 \\
CAMK & 74 & 58 & 28 & 48 \\
CK1 & 12 & 8 & 3 & 38 \\
CMGC & 64 & 60 & 37 & 62 \\
Lipid & 20 & 13 & 10 & 77 \\
Other & 81 & 51 & 26 & 51 \\
STE & 47 & 81 & 13 & 67 \\
TK & 90 & 35 & 54 & 54 \\
TKL & 43 & 401 & 19 & 54 \\
\hline Total & 215 & & 528 &
\end{tabular}

For inclusion in KCGS, an inhibitor was selected if the DiscoverX assay panel showed $\mathrm{K}_{\mathrm{D}}<100 \mathrm{nM}$ on its target kinase and $\mathrm{S}_{10}(1 \mu \mathrm{M})<0.025$ in a full panel kinase screen [16]. 
For inhibitors from PKIS, the assay data from the Nanosyn screening panel of 230 kinases was used to calculate the selectivity index in lieu of submitting the compounds to scanMAX. For inhibitors from PKIS2 and the newly donated compounds, the data from the DiscoverX scanMAX panel of 401 kinases was used to calculate the selectivity index. Compounds that met the inclusion criteria were manually triaged to maximize the coverage of the human kinome. Our aspirational goal was to include two unique chemotypes for each kinase and care was taken not to over-represent kinases that had been more heavily studied (such as EGFR, MAPK14, and GSK3B). For the poorly studied dark kinases, there was often only one or two compounds to select that met the inclusion criteria. Finally, in those cases where two compounds had equivalent kinase profiles, preference was given to inclusion of the chemotype with fewer exemplars in the set. Using these guidelines, version 1.0 of KCGS was assembled with a total of 187 kinase inhibitors. Summary information for each inhibitor is contained in Table S1 and the full kinase profiles can be accessed at www.randomactsofkinase.org.

\subsection{Kinase Coverage}

The set covered a total of 215 human kinases, which was more than $50 \%$ of the full scanMAX assay panel (Tables 1 and S2). Across the branches of the kinome, broad coverage was found in the TK family (67\%) and CMGC family (62\%). While KCGS appears to cover $71 \%$ of the atypical kinases, only a small fraction of these kinases have assays in the scanMAX panel. Lower coverage was obtained for the CK1 (38\%) and STE (31\%) families. In total, 114 kinases were covered by two or more inhibitors, while the remaining 98 kinases have only one useful inhibitor in the current set (Table S1). Ideally, every kinase would be covered by inhibitors from multiple chemotypes to aid analysis of phenotypic screening data. This remains a goal for future expansion of the set.

Despite the tractability of kinases as drug targets, the majority of the kinome is poorly annotated and remains dark with respect to its role in human biology, in part due to a paucity of reagents. The NIH IDG initiative has nominated 162 dark kinases (Figure 1 and Table S2) for development of chemical and biological tools in an effort to seed research on these understudied proteins [7]. KCGS contains inhibitors of 37 of the IDG dark kinases (Table S2), which may be useful as initial chemical tools to study these kinases. These KCGS compounds can also be used as starting points for development of high-quality chemical probes as the biological function of their kinase targets becomes better understood and the investment in additional optimization is warranted. We utilized a recent data set $[24,25]$ annotated to human protein-coding genes and their genetic relevance to look at the frequency of PubMed publications on individual kinases. Figure 1 depicts the publication counts for kinases covered by KCGS. The set contains inhibitors of most of the more highly studied (top 25\%) kinases, but importantly it also contains inhibitors that cover some of the darkest kinases. Future expansion of KCGS will focus on filling the gaps in coverage of the dark kinases.

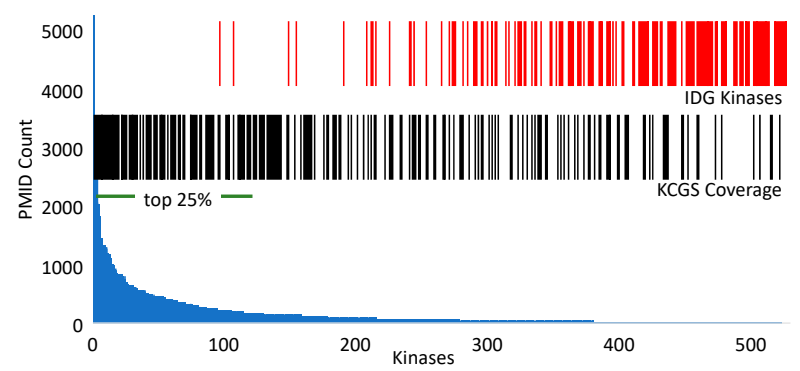

Figure 1. Analysis of kinase coverage in KCGS by publication frequency. The blue histogram indicates the number of publications for each kinase in PubMed (Table S2) [24,25] ordered by frequency with the top $25 \%$ noted by the horizontal bar. The red vertical bars indicate the 162 poorly studied dark kinases nominated by the NIH Illuminating the Druggable Genome (IDG) initiative [7]. The black vertical bars indicate the 215 kinases covered by an inhibitor in KCGS version 1. 


\subsection{Chemotype Analysis}

To aid analysis of screening data and to support future expansion of KCGS, a method was developed to assign each inhibitor to a specific chemotype based on the chemical structure of its hinge-binding moiety. To accomplish this, 119 known kinase hinge-binder substructures were defined manually and codified using SMILES (Simplified Molecular Input Line Entry System) arbitrary target specification (SMARTS) [26]. To resolve issues where an inhibitor could be assigned to multiple bins, SMARTS were given a priority order. Kinase inhibitors that lacked an obvious hinge-binding group were grouped separately into an additional SMARTS bin. Applying this analysis to the 187 KCGS inhibitors, the compounds were found to occupy 67 of the 120 SMARTS bins (Figure 2A and Table S3). Nine of the bins contained six or more inhibitors, 27 bins had two to five members, and 31 bins contained only one exemplar. The nine most highly populated SMARTS bins contain well-known kinase inhibitor scaffolds, such as indazoles, oxindoles, quinazolines, quinolines, and pyrimidines. Six KCGS compounds that lack an obvious hinge-binder group were placed in the "other" bin. They include an allosteric PAK inhibitor and two allosteric MEK inhibitors. For bins containing multiple exemplars, the individual inhibitors often showed activity on kinases located in several different branches of the kinome. For example, the 13 oxindoles in KCGS showed a cluster of activity on CMGC kinases, but they also inhibited TK, TKL, and STE kinases (Figure 2B). While the oxindole chemotype has been found in many highly promiscuous kinase inhibitors, the inclusion of several oxindoles in KCGS demonstrated that this chemotype can also produce highly selective kinase inhibitors by judicious optimization of the molecules. KCGS contained nine exemplars in the 4-anilino-quinazolines bin (Figure 2C). Six FDA-approved kinase inhibitors that target EGFR and ERBB2 also fall into this bin. However, the SMARTS analysis highlights that modification of the 4-anilino-quinazoline chemotype can also generate inhibitors with activity on several adjacent kinase subfamilies.

A.<smiles>C=CC</smiles>

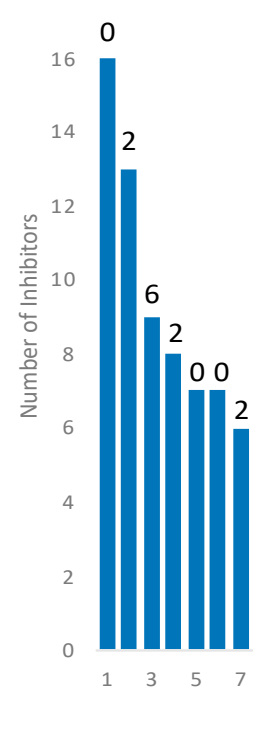

KCGS Chemotype Bin

\begin{tabular}{|cl|}
\hline Bin \# & Chem otype \\
\hline 1 & 6-Ar-indazole \\
2 & oxindole \\
3 & 4 -anilino-quinazoline \\
4 & fused amino-pyrimidine \\
5 & 2-amino-pyrimido-diazepin-6-one \\
6 & 4-Ar-pyridine \\
7 & 4-anilino-quinoline \\
8 & 2-anilino-4-aryl-pyrimidine \\
9 & other \\
\hline
\end{tabular}

Figure 2. Cont. 
B.

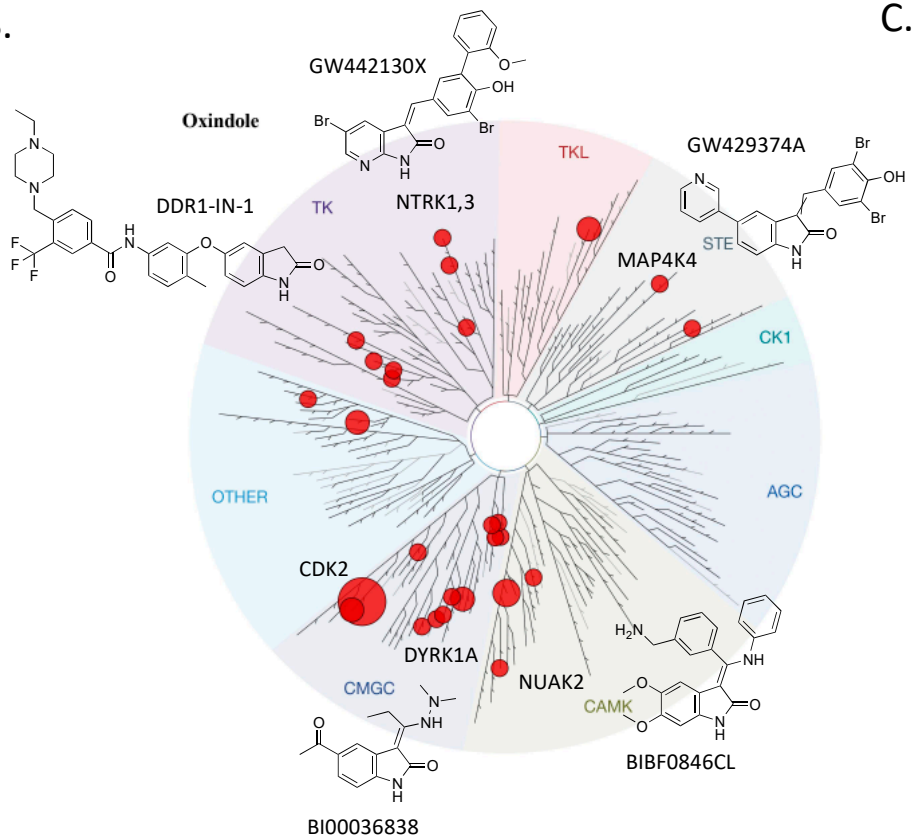

C.

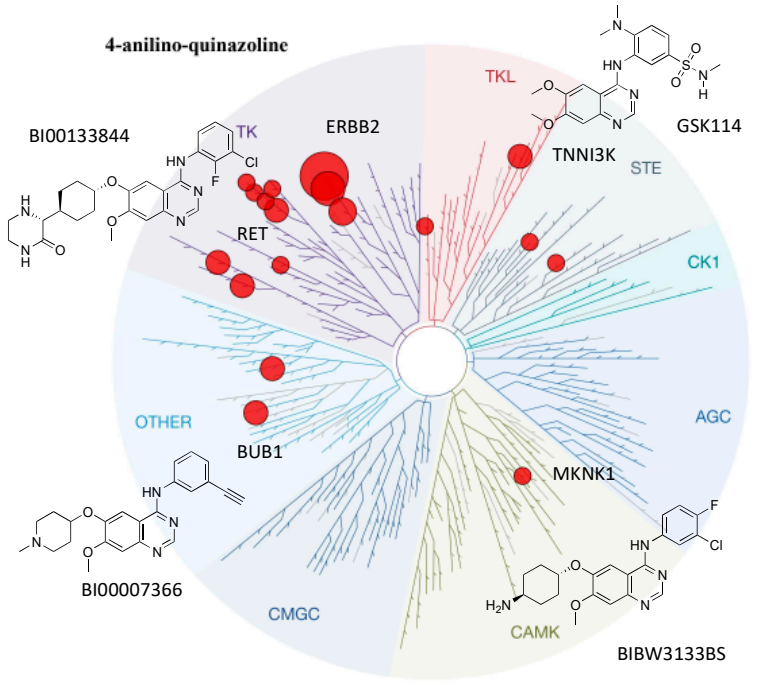

Figure 2. KCGS contains inhibitors from 67 distinct chemotypes. (A) This graph displays the number of inhibitors in each SMILES arbitrary target specification (SMARTS) bin. The numbers above the left-most 9 bars represent the number of FDA approved kinase inhibitors in these chemotype bins at the time the manuscript was written. The inset provides names of the 9 most highly populated bins. (B) Tree plot of the human kinases with each subfamily uniquely shaded. Kinases covered by a member of the oxindole SMARTS bin are displayed as red dots, scaled by the number of compounds inhibiting a specific kinase. Representative chemical structures form the oxindole SMARTS bin are shown. (C) Kinases covered by the 4-anilino-quinazoline SMARTS bin with representative chemical structures. (\# = number).

\subsection{Calculated Properties}

All of the inhibitors in KCGS were originally the product of medicinal chemistry projects to target specific kinases. As such, many of them had been optimized with an eye on physicochemical properties and cellular activity. To evaluate the overall quality of the set, the calculated properties of each inhibitor from KCGS were determined in SwissADME [27] and compared to a set of 52 FDA-approved kinase inhibitors [28]. SMILES strings representing each inhibitor were input into SwissADME to generate the predicted solubility and calculated lipophilicity (Table S1). For predicted solubility, the inhibitors were binned into four categories ranging from poorly to very soluble. The solubility profile of both inhibitor sets was similar (Figure 3A). The majority of compounds were predicted to be moderately soluble or better for both KCGS (88\%) and the FDA-approved inhibitors $(78 \%) . \log \mathrm{P}$ is a common measure of lipophilicity and is considered a critical factor in assessing the drug-like properties of small molecules [29]. The SwissADME consensus $\log \mathrm{P}(\mathrm{cLog} P)$, which is the arithmetic mean of five calculated values (XLOGP3, WLOGP, MLOGP, SILICOS-IT, and iLOGP), was used to compare KCGS to the FDA-approved kinase inhibitors. The results showed that KCGS clogP values trended towards lower lipophilicity than the FDA-approved drugs, with $65 \%$ of the KCGS inhibitors falling between cLogP 2 and 4 and proportionally fewer inhibitors with cLogP $>4$ (Figure 3B). Overall, these calculations support the premise that the inhibitors in KCGS have physical properties that render them well-suited for use in cell-based assays. 
A

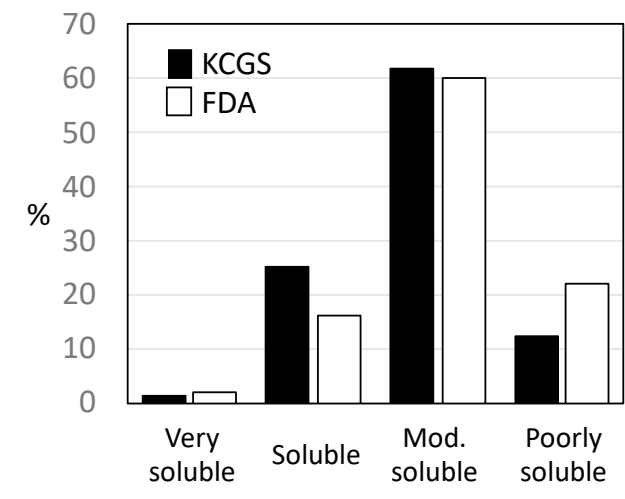

B

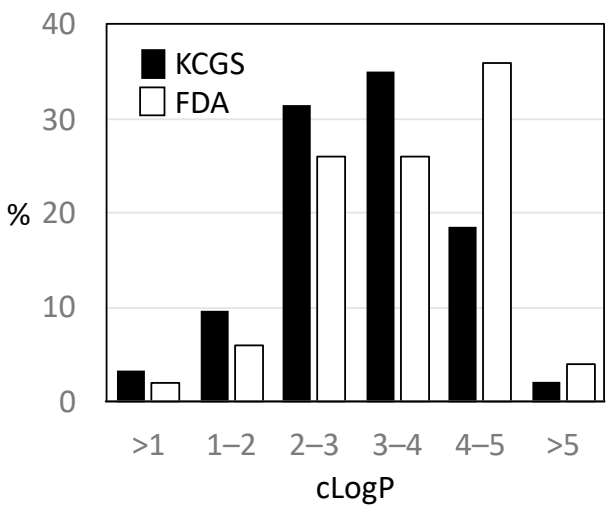

Figure 3. Calculated properties. (A) Predicted solubility of the KCGS compounds (black) compared to 52 FDA-approved kinase inhibitors (white) split into four categories and shown as percentage of the set. (B) Hydrophobicity analysis of the KCGS compounds (black) using SwissADME compared to the FDA-approved kinase inhibitors (white) split into six ranges of cLogP and shown as percentage of the set.

\subsection{Chemogenomic Screening}

To format KCGS for distribution to a large number of researchers, $10 \mathrm{mM}$ DMSO stock solutions of the 187 inhibitors were aliquoted into 384 -well format plates. Then, $1 \mu \mathrm{L}$ of each inhibitor was dispensed to each well of the plate using an Echo 550 acoustic dispenser for accurate delivery. This $1 \mu \mathrm{L} / 10 \mathrm{mM}$ volume provides sufficient compound to run 100 assays at a $1 \mu \mathrm{M}$ inhibitor concentration in 96-well format and 200 assays in a 384-well format (assuming 100 and $50 \mu \mathrm{L}$ working volume, respectively, see Supplementary File S2). KCGS was delivered with a plate map that delineates compound identification numbers as well as the kinase profile for each compound (Table S1). Based on the kinase selectivity profile of the inhibitors, $1 \mu \mathrm{M}$ is the recommended screening concentration for chemogenomic experiments to support hit identification and target deconvolution. Screening at higher concentrations will likely complicate data interpretation due to additional undocumented off-target activity of the inhibitors. To aid with hit follow-up, additional quantities of each inhibitor were available from the SGC-UNC for full-dose response and secondary assays.

\subsection{Cell Toxicity}

To facilitate the use of KCGS in cell-based assays, we determined the acute toxicity of the individual inhibitors at a high dose $(10 \mu \mathrm{M})$ in HeLa cells. After $24 \mathrm{~h}$ treatment, high content imaging [30,31] was used to measure healthy cell count as well as the percent of necrotic and apoptotic cells, which identified those inhibitors that exhibited varying degrees of toxicity (Figure 4 and Table S4). In total, 134 of the kinase inhibitors had little or no effect on total cell count. A total of 43 of the inhibitors reduced cell count by $20 \%$ or more. The most toxic compounds that decreased cell count by $>67 \%$ are highlighted in Figure $4 \mathrm{~A}$. The cell toxicity displayed by these compounds may be due to their inhibition of kinases that affect cell division or cell viability, either as a primary or secondary target. Among the compounds with the largest effect on cell count were inhibitors of kinases involved in cell cycle progression, checkpoint regulation, and cell division including the CDKs (GW416981X, THZ531, BI00036838), AURKC (GW814408X), ATR (VE-822), and CHK1/2 (CCT244747, CCT241533). Other compounds with significant toxicities in HeLa cells at the $10 \mu \mathrm{M}$ dose were GW683134A, a type II inhibitor of KDR, KIT and TEK, and PFE-PKIS 29 , a very potent $(<10 \mathrm{nM})$ inhibitor of $\mathrm{mTOR}$ and several lipid kinases including all isoforms of PI3K. 

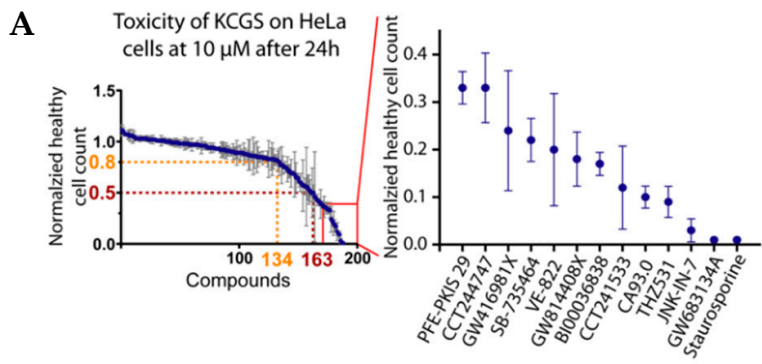

B

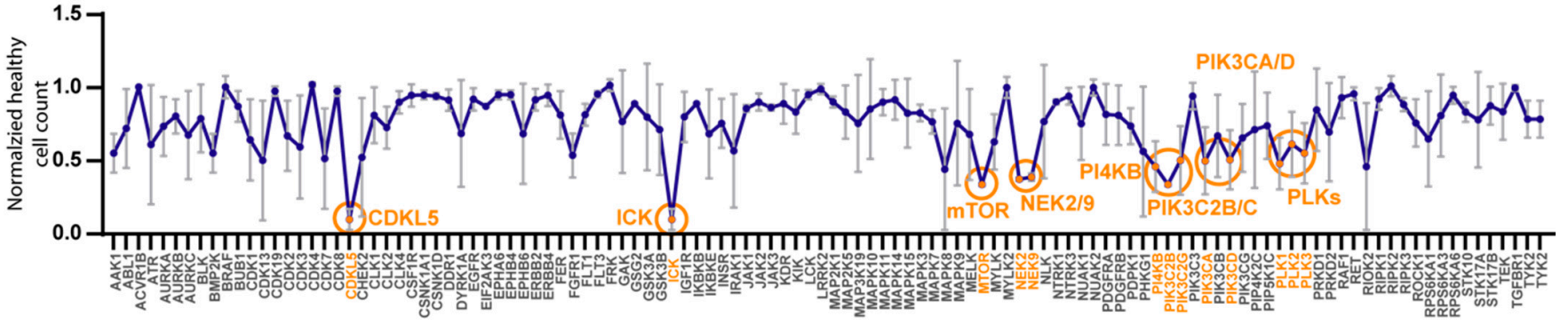

Figure 4. Cell toxicity assessment. (A) Effects of KCGS compounds at $10 \mu \mathrm{M}$ on HeLa cells after $24 \mathrm{~h}$. Measurements were made in triplicate with standard errors shown. Shown is the normalized healthy cell count with highlighted thresholds of 0.8 (80\% healthy cells) and 0.5 (50\% healthy cells). The panel on the right side displays the compounds with the greatest effect on healthy cell count in HeLa cells. (B) Averaged toxicity measured by normalized healthy cell count for every target covered by two or more chemotypes. Highlighted are target kinases that show a significantly lower healthy cell count than the DMSO control.

To gain further insight into kinases associated with cell toxicity, we performed an analysis of every kinase that is covered in KCGS by two or more distinct chemotypes (Figure 4B). Several kinases were identified whose inhibition resulted in a significantly lower healthy cell count. Several inhibitors of the polo-like kinases (PLKs) showed toxicity, as did inhibitors of the PIK3C and PI4KB lipid kinases. The apparent toxicity of NEK2/NEK9 inhibition by GSK579289, GSK461364, GSK579289, and GSK237701 may also be attributed to the collateral PLK inhibition of these compounds at the high concentration that the assay was performed at. The apparent toxicity of inhibition of the dark kinases CDKL5 and ICK by JNK-IN-7 and BI00036838 may also be due to the inhibition of the other kinase targets of these two inhibitors (Table S1).

\subsection{Cell Growth}

To further document the effect of KGCS on cell viability, we performed assays for cell growth [32] in 16 immortalized cell lines that were selected to cover breast, ovarian, prostate, colorectal, lung, skin, brain, and pancreatic cancers (Table S5). Nonmalignant breast and lung cell lines were included for comparison. Using a $1 \mu \mathrm{L}$ aliquot of KCGS (10 mM in DMSO), the set was screened in duplicate across the 18 cell lines at a compound concentration of $1 \mu \mathrm{M}$. The effects on cell growth, viability and cell cycle were determined after $72 \mathrm{~h}$ treatment using high-throughput microscopy [33]. Growth rate (GR) inhibition values, employed to account for variable division times, were computed [34]. GR values below zero are indicative of net cell loss whereas values between zero and one can result from growth arrest or a combination of cell death and proliferation over the assay duration. Therefore, the fraction of dead cells and the cell cycle distribution of the live cells were determined (Table S6). One cell line (SW1783) did not grow under the assay conditions and was excluded from analyses (Table S6). The effect of KCGS across the remaining 17 cell lines is depicted in Figure 5. 


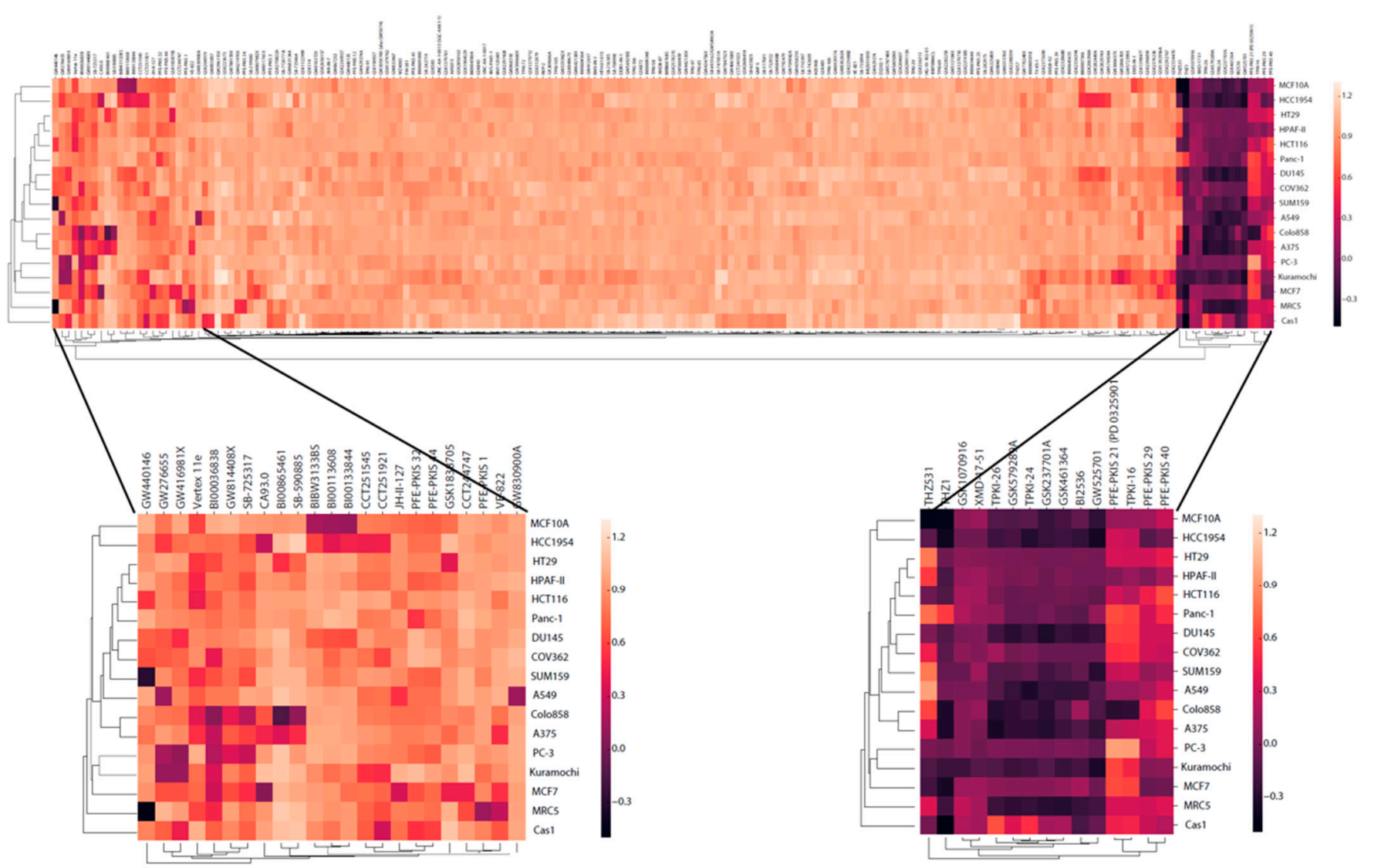

Figure 5. The kinase inhibitors in KCGS have different effects on cell growth across a wide range of lines. Normalized growth rate (GR) inhibition values were determined at $72 \mathrm{~h}$ post compound treatment. Each row displays data from a single cell line. Each column displays data from a single compound. GR is colored from -0.5 in black to 1.3 in yellow. The expanded views display data for compounds that showed cell-line selective decrease in GR and relatively cell-line independent effects, respectively.

Analysis of GR across the 17 cell lines identified three categories of kinase inhibitors. The first category included the majority of inhibitors in KCGS that showed no discernable effect on cell growth, with GR values within $10 \%$ of the DMSO control. The second category containing 15 inhibitors showed a $>30 \%$ decrease in GR across most of the cell lines. Six of these compounds (TPKI-24, TPKI-26, GSK461364, GSK579289A, GSK237701A, and BI2536) have PLK inhibitory activity, two are allosteric MEK inhibitors (PFE-PKIS 21 and TPKI-16), and two are inhibitors of aurora kinases (XMD-17-51 and GSK1070916). Notably, only two of these inhibitors (THZ531 and PFE-PKIS 29) were shown to be cytotoxic at a higher concentration in the HeLa cell experiment. The third category contained 23 inhibitors that showed cell line-dependent effects on GR. Six of these compounds (GW416981, BI00036838, GW814408X, SGK-GAK-1 (CA93.0), CCT244747, and VE-882) have been identified as toxic to HeLa cells, but when tested at $1 \mu \mathrm{M}$ across a wider range of lines their effects were now shown to be dependent on other cellular factors and not intrinsic to the compounds alone. Based on the annotation of these compounds, inhibition of several kinases was highlighted as being responsible for cell line-dependent effects. These kinases include multiple CDK isozymes, GAK, BRAF, and BLK. Determination of whether selective inhibition of these kinases would have potential therapeutic utility in specific cancers will require confirmatory follow-up studies such as CRISPR dropout screens and screening of alternate inhibitors of the same targets. However, these data highlight the power of screening a chemically and biologically diverse chemogenomic set of kinase inhibitors to determine how they perturb a simple cell phenotype. 


\subsection{Kinases Linked to Autophagy}

Autophagy is a central mechanism that helps maintain cellular homeostasis. Autophagy is activated in response to different stress conditions such as starvation, protein aggregation, oxidative stress, bacterial infection, inhibition of the TOR1 pathway, and others $[35,36]$. To determine the effect of kinases on autophagic flux, the KCGS library was screened at $1 \mu \mathrm{M}$ concentration in RPE1 cells stably expressing the autophagic flux reporter construct GFP-LC3B-RFP-LC3B $\Delta$ C [37] (Figure 6). The cells were monitored in a time-dependent manner so that the ratio of GFP/RFP intensity ratio represented the level of autophagic flux. The averaged GFP/RFP ratio was subsequently normalized to time point $0 \mathrm{~h}$ in order to facilitate easy visualization of differences to the autophagy control compounds Torin1 (inducer) and Torin1 plus Bafilomycin A (inhibitor and deacidifier of lysosomes) compared to the DMSO vehicle control (Table S7).

A

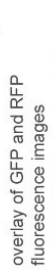

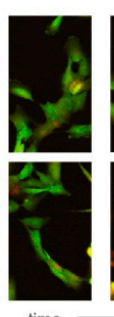
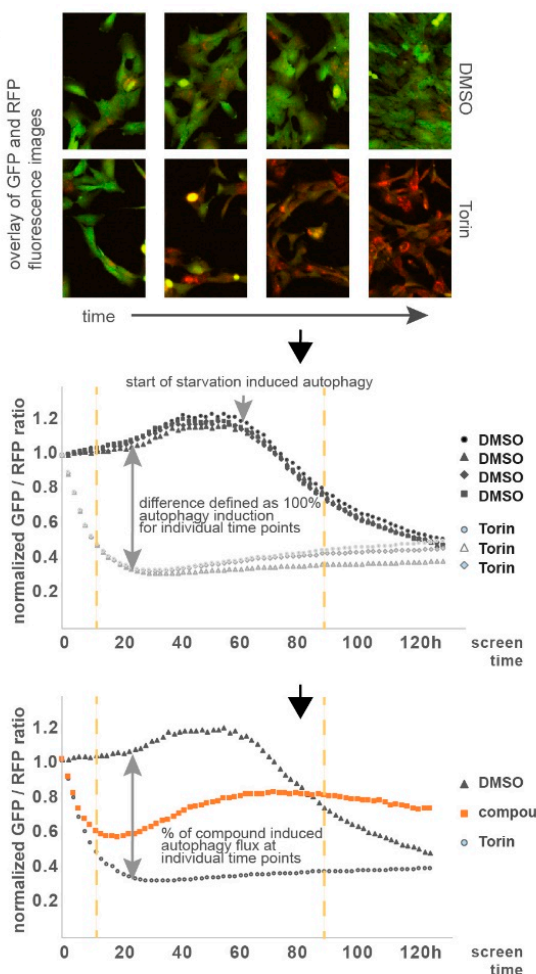

B

\begin{tabular}{|c|c|c|c|c|}
\hline & \# of hits & $\begin{array}{c}\text { Proliferation } \\
\text { rate }\end{array}$ & $\begin{array}{c}\text { Cell } \\
\text { apprearance }\end{array}$ & $\begin{array}{l}\text { Autophagy } \\
\text { flux }\end{array}$ \\
\hline category 1 & 6 & normal & healthy & $\begin{array}{c}\text { partly } \\
\text { increasing }\end{array}$ \\
\hline category 2 & 6 & normal & healthy & $\begin{array}{c}\text { partly } \\
\text { decreasing }\end{array}$ \\
\hline category 3 & 5 & delayed & healthy & $\begin{array}{l}\text { increasing } \\
\text { over time }\end{array}$ \\
\hline category 4 & 2 & delayed & healthy & $\begin{array}{c}\text { decreasing } \\
\text { over time }\end{array}$ \\
\hline category 5 & 1 & delayed & healthy & $\begin{array}{l}\text { increased \& } \\
\text { dropping }\end{array}$ \\
\hline category 6 & 8 & none & sick / dead & $\begin{array}{c}\text { partly } \\
\text { increased }\end{array}$ \\
\hline
\end{tabular}
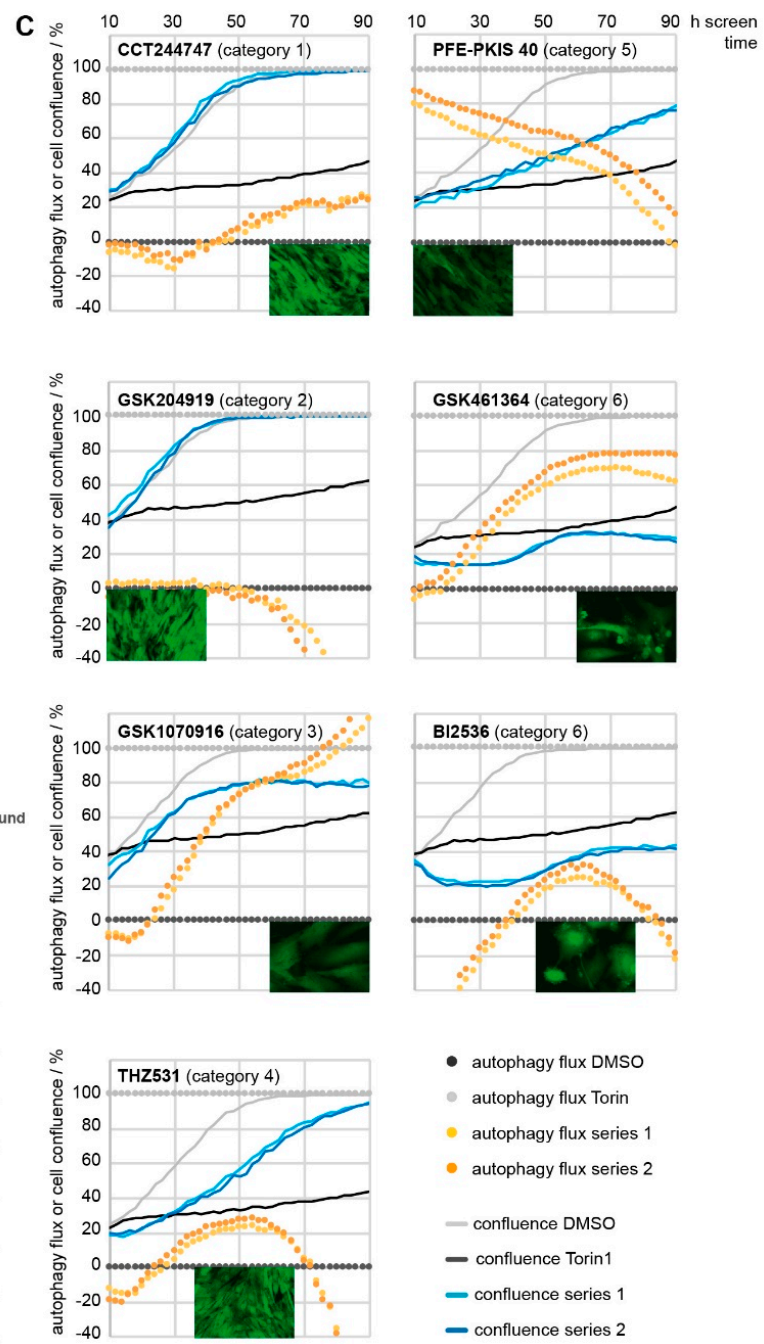

Figure 6. Autophagic flux assay. Compounds $(1 \mu \mathrm{M})$ were analyzed for their effect on autophagy flux in RPE1 cells stably expressing the general autophagy flux reporter GFP-LC3B-RFP-LC3 $\Delta$ C. Phase contrast as well as fluorescent images (GFP, RFP) were taken at indicated time points. (A) Ratio of the GFP/RFP signal correlating with high (Torin1; defined as $100 \%$ ) and low (DMSO; defined as $0 \%$ ) autophagy flux. Compound-induced changes in autophagic flux are represented as percentage of this difference. Small black arrows indicate assay workflow (B) Hits are defined as compounds showing > $20 \%$ aberration of the GFP/RFP ratio compared in five or more consecutive time points and categorized according to their cell proliferation rate as well as visual appearance. $(\#=$ number $)(C)$ Examples of compounds with an effect on autophagic flux. Cell confluence is presented as percentage of covered growth area. Visual appearance of cells and cell confluence were ascertained by examining fluorescent images of the GFP channel at $96 \mathrm{~h}$ screen time. Graphs contain the individual average of experiments run in triplicate at two different times. 
As expected, DMSO-treated cells had a stable level of autophagic flux until nutrients in the media were depleted and they entered a starvation-induced phase of autophagy induction. In contrast, the GFP/RFP ratio of Torin1-treated cells rapidly decreased within the first screening hours and stayed low over the complete time period of the five-day screening. Of note, treatment with Torin I caused an arrest of cell proliferation but no cell death. Confluence analysis was based on phase contrast images corresponding to the cell-covered area of each well. In this assay, cell health can only be assessed based on visual appearance of the cells and cell proliferation.

Hits were defined as compounds that showed $>20 \%$ aberration of the GFP/RFP ratio in at least five or more consecutive time points, equivalent to a $10 \mathrm{~h}$ assay window. Hits were grouped in six categories based on the increase or decrease in proliferation rate, cell appearance and autophagy flux, respectively (detailed in Figure 6). Category 1 hits included GW416981X, a potent CDK1-3 inhibitor. The CDK inhibitors roscovitine and purvalanol have previously been shown to induce autophagy [38]. Likewise, CHK1 inhibition, represented by the category 1 hit CCT244747, has previously been linked to autophagy induction [39]. However, we also identified several new potential kinase targets for autophagy. For example, GSK204919, a potent dual PRKD1/2 inhibitor, caused a reduction in autophagic flux. The role of PRKD in autophagy has not been well-documented. Additional studies are required to link the observed autophagy reduction to PRKD inhibition rather than another kinase target of GSK204919 (e.g., JAK). Categories 3 and 5 compounds contained inhibitors of kinases known to induce autophagy, such as GSK1070916 (an aurora kinase [40] inhibitor) and PFE-PKIS 40 (a PI3K and mTOR [41] inhibitor). Notably, the RPE-1 cells used in the screen did not show any reduction in cell proliferation at $1 \mu \mathrm{M}$ PFE-PKIS 40, despite its toxicity at $10 \mu \mathrm{M}$ in HeLa cells (see above). The behavior of the category 4 compounds THZ531 (CDK inhibitor) and PFE-PKIS 29 (mTOR inhibitor) likely resulted from overlap of autophagy induction with cell toxicity as already identified in the cell health and cell growth assays. Most of the compounds in category 6 have also been identified in the cell growth assays, including several inhibitors with activity on PLK (TPKI-24, TPKI-26, GSK461364, GSK579289A, GSK237701A, and BI2536), a kinase known to regulate both autophagy as well as mitosis [42].

\section{Discussion}

KCGS version 1.0 is currently the best publicly available set of well-annotated potent and selective kinase inhibitors. All of the inhibitors have narrow selectivity profiles as ascertained from screening across an assay panel covering the majority of the human protein kinases. The set can be obtained by any investigator who agrees to the open science principles of not restricting its use by others and also promises to publish the results of their screen (Supplementary File S1). This manuscript describes the chemical structure and kinase annotation of all of the inhibitors in the current set. We recognize that there is additional room for improvement in the breadth (more kinases) and depth (more chemotypes per kinase) of kinase coverage and in the biological annotation of the set. However, initial characterization of KCGS in phenotypic screens confirmed the utility of the set for chemogenomic exploration of kinase signaling. Screening across 18 cell lines identified a subset of compounds that selectively inhibit their growth. Some of these compounds point to dark kinases that have received little attention as potential drug targets. A screen for autophagy uncovered additional kinase pathways that warrant further exploration. The narrow spectrum kinase activity of the individual inhibitors and the accompanying annotation supports rapid identification of target kinases for additional studies. While the compounds are generally nontoxic, we recommend that KGCS is screened at a maximum concentration of $1 \mu \mathrm{M}$ in cells to minimize the potential for inhibition of additional kinases or off-target toxicity.

Several ongoing activities will support KCGS, which remains as the best publicly available set of kinase inhibitors. One such activity was obtaining screening data on all KCGS compounds in the same assay format. This would ensure that results were 
comparable and offers the possibility of providing new kinase coverage. With about a quarter of the set originating in PKIS, which was only screened at Nanosyn, we had an opportunity to further profile these compounds in another 200 kinase assays by utilizing DiscoverX scanMAX. This screening was recently performed at a screening concentration of $1 \mu \mathrm{M}$ (Table S8) for direct comparison to previously published data [15]. For some compounds, we identified new kinase binding partners in the additional assays while for others we did not. For example, GSK270822A and GSK299115A, both amino indazoles, were previously identified as ROCK1 inhibitors when profiled at Nanosyn [15]. When screened more broadly, these compounds were also found to bind to ROCK2, JAK2, JAK3, TYK2, NUAK2, and LATS2 $\geq 25 \mathrm{PoC}$. All these results need to be verified as true positives via Kd determination and this work is ongoing. The addition of ROCK2 is perhaps not surprising due to its homology to ROCK1. The inhibition of NUAK2, if confirmed, provides another chemotype to use to study the biology of this understudied kinase. GW814408X is a KCGS compound that based on original PKIS data only demonstrated potent binding to a single kinase (AURKC). Upon further screening, this compound was found to inhibit a number of other kinases. We will determine Kd values for new kinase hits for all KCGS version 1.0 compounds discovered with this new screening. If compounds fall outside our desired selectivity window, they will be replaced in future releases.

Currently, $51 \%$ of the screenable kinome, as defined by the DiscoverX scanMAX, is covered by KCGS version 1.0 for a total of 215 human kinases. Over 100 of these kinases were selectively inhibited by two or more chemotypes in the set. Our originally stated goal was to cover all human kinases with two or more chemotypes, so additional inhibitors are still required for those kinases that are covered by only single chemotype. There are an additional 250 "gap kinases" where we are still seeking an inhibitor that meets our minimal potency and selectivity criteria for inclusion in the set. For many of the gap kinases that are routinely screened in the DiscoverX scanMAX, identification of a nonpromiscuous inhibitor is the primary challenge but may be achievable through iterative medicinal chemistry to improve selectivity. Additionally, there are over 50 human kinases for which robust biochemical screening assays are not readily accessible in any format. For these kinases, it is not yet known if useful inhibitors already exist in the current set or among molecules that are in the public domain. Many of these dark kinases are difficult to express and purify or represent pseudokinases with little or no catalytic activity. Development of new screening formats or assay methodologies will be required to identify a complete set of inhibitors for the whole kinome.

One limitation to the design and selection of the inhibitors in KCGS was the use of potency and selectivity data from cell-free biochemical assays. The activity of kinase inhibitors in cells can be affected the binding of other cellular components to the kinase. In addition, some inhibitors may be less potent in cells if they are not efficient at crossing the cell membrane. However, the provenance of compounds included in KCGS, either from lead optimization programs in the pharmaceutical industry or the product of academic chemical probe development projects, suggests that most of them are likely to be cell active. In fact, the profile of physical properties across KCGS is as good if not better than a set of 52 FDA-approved kinase inhibitor drugs. Regardless, it is not uncommon for kinase inhibitors to demonstrate lower potency in cells than in cell-free assays. A recent advance in the application of NanoBRET technology to measure the target engagement of kinase inhibitors in living cells has provided a method to study this issue [43]. NanoBRET assays have now been developed for 133 of the human kinases that are inhibited by the molecules in KCGS version 1.0. By using these NanoBRET assays, we have begun the process of annotating each of the inhibitors in KCGS for its activity against its corresponding target kinase in live cells. These data will aid the deconvolution of phenotypic screening data of KCGS and identify kinases for which inhibitors with improved cell activities will be required in future releases.

The inhibitors in KCGS were sourced from multiple industrial and academic laboratories in a conscientious effort to maximize both the number of chemotypes (chemical 
diversity) and the breadth of kinase coverage (biological diversity). While the core of the set is still composed of molecules that were published by GlaxoSmithKline chemists, KCGS version 1.0 contains inhibitors that originated from the laboratories of four pharmaceutical companies and three academic laboratories. We continue to seek new inhibitors to add to KCGS that represent either a new chemotype or an inhibitor of a gap kinase. To this end, we have completed profiling of molecules that have been donated by three additional companies as well as molecules synthesized in our laboratories and by academic collaborators. Inhibitors representing new chemotypes that increase the depth of coverage on many kinases will be made available as a supplemental set (KCGS version 1.1). Identification of potent and selective inhibitors of the gap kinases represents a more formidable yet surmountable challenge. We continue to welcome donations of candidate inhibitors of these kinases from industrial and academic laboratories to support expansion of the KCGS. All donor laboratories, in return, receive copies of the full KCGS set and the satisfaction of contributing to the goal of maintaining the best publicly available set of a high-quality kinase inhibitors.

\section{Materials and Methods}

\subsection{KCGS}

The current version of KCGS is available in $1 \mu \mathrm{L}$ aliquots of a $10 \mathrm{mM}$ DMSO solution at www.sgc-unc.org/request-kcgs/.

\subsection{Kinase Assays}

Compounds were screened at $1 \mu \mathrm{M}$ using the KINOMEscan technology in the scanMAX assay panel of 401 wild-type human kinases and $S_{10}$ was calculated as previously described [16]. Compounds with $S_{10}<0.04$ were submitted for $K_{D}$ measurement on kinases with POC $<20 \%$. Compounds with $\mathrm{K}_{\mathrm{D}}<100 \mathrm{nM}$ and $\mathrm{S}_{10}(1 \mu \mathrm{M})<0.025$ were selected as candidates for inclusion in KCGS.

\subsection{Chemotype Binning}

Each molecular substructure or bin representing the desired hinge-binder was manually codified in SMARTS language [26] and were given a priority order. Each molecule in KCGS was represented as a SMILES code. The SMARTS search was performed using Open Babel [44] to generate an .smi file to associate the SMILES code of each molecule with a specific SMARTS bin. All .smi files were processed in MATLAB [45] to create a compound-SMART matrix. Compounds with multiple matches were assigned to bin that corresponded to the highest priority SMARTS.

\subsection{Cytotoxicity Assays}

A triple staining high content screen was performed as previously described [30,31]. HeLa cells exposed to KCGS compounds at $10 \mu \mathrm{M}$ for $24 \mathrm{~h}$ were stained with the three dyes: Hoechst $33342(1 \mu \mathrm{M})$, Yo-Pro $3(1 \mu \mathrm{M})$ and Annexin V $(0.3 \mu \mathrm{L}$ per well) for $1 \mathrm{~h}$. Cellular fluorescence was measured using the CQ1 high content imaging system (Yokogawa, Sugarland, TX, USA) with the following setup parameters: Brightfield transmitted light at $70 \%$ for $50 \mathrm{~ms}$; Hoechst 33342 was excited by $100 \mathrm{~ms}$ exposure; Ex $405 \mathrm{~nm} / \mathrm{Em} 447 / 60 \mathrm{~nm}$, Yo-Pro 3 by $100 \mathrm{~ms}$ exposure; Ex $561 \mathrm{~nm} / \mathrm{Em}$ 617/73 nm and Annexin V (Alexa 488, Ther-

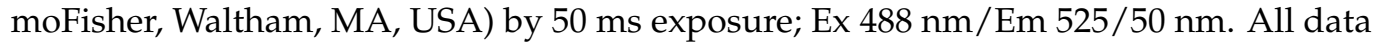
were analyzed by the Pathfinder software and four categories for cells were designated: healthy cells, early apoptosis, late apoptosis, and necrosis. Each category was calculated as a percentage for every inhibitor. Cell nuclei were classified as either healthy, pyknosed, or fragmented.

\subsection{Cell Growth Assays}

The KCGS library compounds were arrayed in a 384-well plate at a concentration of $1 \mathrm{mM}$. Four breast cell lines, (SUM159, MCF7, MCF10A (nonmalignant), and HCC1954), 
and two each of ovarian (COV362 and KURAMOCHI), prostate (PC-3 and DU145), colorectal (HT29 and HCT116), lung (A549 and MRC-5), melanoma (COLO858 and A375), glioblastoma (Cas1 and SW1783), and pancreatic (Panc-1 and HPAF-II) cancer cell lines were maintained in their recommended growth media at $37^{\circ} \mathrm{C}$ in $5 \% \mathrm{CO}_{2}$, and were seeded in 384-well CellCarrier plates (Perkin Elmer, Waltham, MA, USA) at the densities listed in Table S5. Cells were allowed to adhere for $24 \mathrm{~h}$ and treated in duplicate with the KCGS library by pin transfer for a final concentration of $1 \mu \mathrm{M}$. Cells were stained and fixed at the time of pin transfer and following $72 \mathrm{~h}$ of treatment. Cells were pulsed for one hour with EdU (Lumiprobe, Hunt Valley, MD, USA) and stained with 1:2000 LIVE/DEAD Far Red Dead Cell Stain (LDR) (Thermo Fisher Scientific, Waltham, MA, USA). Cells were then fixed with 3.7\% formaldehyde (Sigma Aldrich, St. Louis, MO, USA) for $30 \mathrm{~min}$ and permeabilized with $0.5 \%$ Triton X-100 in PBS. The EdU was labeled with cy3-azide (Lumiprobe, Hunt Valley, MD, USA) for $30 \mathrm{~min}$. The cells were then blocked for one hour with Odyssey blocking buffer (LI-COR, Lincoln, NE, USA), and stained overnight at $4{ }^{\circ} \mathrm{C}$ with $2 \mu \mathrm{g} / \mathrm{mL}$ Hoechst 33342 (Sigma Aldrich, St. Louis, MO, USA) and a 1:1000 dilution of anti-phospho-histone H3 (pHH3) Alexa 488 (Ser10, clone D2C8) conjugated antibody (Cell Signaling Technologies, Danvers, MA, USA). Fixed cells were imaged with a 10x objective using an IXM-C microscope and analyzed using MetaXpress software (Molecular Devices, San Jose, CA, USA). Nuclei were segmented based on their Hoechst signals. DNA content, defined by the total Hoechst intensity within the nuclear mask, was used to identify cells in the G1 and G2 phases of the cell cycle. The average LDR, EdU and phospho-histone $\mathrm{H} 3$ intensities within the nuclear masks were determined and used to classify cells as dead, in S phase or in M phase, respectively. Cells with intermediate DNA content and no EdU signal were classified as $S$ phase dropout cells. Live cell counts were normalized to DMSO-treated controls on the same plates to yield normalized growth rate inhibition (GR) values as described previously [32].

\subsection{Autophagy Assays}

RPE1 cells (1500 cells/well in $50 \mu \mathrm{L}$ DMEM/F12, 10\% FBS, and 1\% P/S) stably expressing the GFP-LC3-RFP-LC3 $\triangle$ C autophagy flux reporter [37] were seeded in 384-well plates and grown for approximately $18 \mathrm{~h}$. In total, $50 \mu \mathrm{L}$ media with $2 \times$ compound concentration were added and plates were subsequently placed and monitored in an IncuCyte ${ }^{\circledR}$ (Sartorius, Bohemia, NY, USA) instrument. Cells were scanned at indicated times for phase contrast and fluorescence intensity (GFP and RFP) to obtain information about confluence and autophagic flux, respectively. Hits are defined as compounds showing $>20 \%$ aberration of the GFP/RFP ratio compared to the control compounds DMSO (0.1\%) and Torin1 $(250 \mathrm{nM})$ in at least 5 or more consecutive time points (equivalent to $10 \mathrm{~h}$ screening time). Compounds were tested in triplicate and the complete screen was performed twice.

\section{Conclusions and Future Directions}

The KCGS is the largest fully annotated set of selective small molecule kinase inhibitors that is accessible to the biomedical scientific community to explore the involvement of kinases in a broad range of human pathologies and cellular pathways. The library is available in an arrayed 384-well format to support phenotypic screening in academic screening facilities and well-equipped laboratories to conduct target identification, mechanistic, synergy, synthetic lethality, and repurposing screens. Biological annotation of a common set of diverse kinase inhibitors will deepen our understanding of the role of kinases in cell signaling and may uncover new targets for drug discovery programs and precursors to new medicines.

Importantly, the set is a key resource supporting the expansion of the druggable genome. For example, kinases have been shown to play a pivotal role in many aspects of cancer physiopathology and have been a highly productive protein family for the treatment of several cancers [46,47]. A highly annotated small molecule library can be employed to comprehensively investigate the role of kinases in cancer biology. Indeed, the 
Target Discovery Institute (TDI), a collaborative cell-based phenotypic screening facility established at the University of Oxford, Nuffield Department of Medicine, to identify more tractable biological targets for potential drug development, has used the KCGS in a range of cancer screens, including combinatorial screens with proteins involved in DNA repair including ATM, SPRTN, FancD2, SETD2, and KMT2D, and pathways involved in ubiquitin-mediated proteolysis, and mRNA dysregulation. The TDI also plans to employ the KCGS library in future combinatorial screens with temazolamide and radiotherapy (ionizing radiation) in glioblastomas. This research has generated its first manuscript from a screen which revealed a striking synthetic lethality between Chk1 inhibition and cyclin F loss [48]. Additionally, several of these ongoing projects have generated very encouraging validated primary hits and, although they will require vigorous follow-up validation, the results highlight the great utility and potential of the KCGC library to uncover novel anticancer targets.

Finally, it is important to acknowledge the potential for employing the KCGS in even more diverse disease-relevant phenotypic screening campaigns across additional human pathologies. Although kinases are heavily targeted in cancer treatment, kinases have also been implicated as causative genes in amyotrophic lateral sclerosis [49], the pathogenesis of Parkinson's Disease [50], and cardiac disfunction [51], to name a few. By providing open access to the KCGS to a diverse range of biomedical research scientists, the potential to accelerate drug target discovery, identify novel kinase mechanisms, and identify kinase vulnerabilities beyond cancer therapeutics is greatly increased.

Supplementary Materials: Supplementary materials can be found at https://www.mdpi.com/1422 $-0067 / 22 / 2 / 566 /$ s1.

Author Contributions: Conceptualization, C.I.W., H.A.-A., D.M.A., A.D.A., P.E., C.F., M.F., S.K., U.L., M.M., S.M., D.O., K.S.S., W.J.Z., T.M.W. and D.H.D.; methodology, C.I.W., W.J.Z., T.M.W. and D.H.D.; software, H.A.-A. and S.V.; validation, C.I.W., I.D., D.E., S.K., S.M. and D.H.D.; formal analysis, C.I.W., I.D., D.E., S.B.H., S.K., C.E.M., S.M., M.S., A.S., M.T., S.V. and D.H.D.; investigation, C.R.M.A., S.B.H., C.E.M., M.S., A.S., S.V. and J.W.; resources, D.M.A., P.E., C.F., M.F., N.S.G., U.L., M.M., D.O., K.S.S. and B.J.T.; data curation, C.I.W., A.D.A., R.E.F., W.J.Z. and D.H.D.; writing-original draft preparation, C.I.W., S.M., S.V., T.M.W. and D.H.D.; writing-review and editing, all authors; visualization, C.I.W., C.E.M., M.S. and D.H.D.; supervision, H.A.-A., I.D., D.E., S.K., S.M., T.M.W. and D.H.D.; project administration, C.I.W., A.P., T.M.W. and D.H.D.; funding acquisition, T.M.W. and D.H.D. All authors have read and agreed to the published version of the manuscript.

Funding: The Structural Genomics Consortium is a registered charity (no: 1097737) that receives funds from AbbVie; Bayer Pharma AG; Boehringer Ingelheim; Canada Foundation for Innovation; Eshelman Institute for Innovation; Genentech, Genome Canada through Ontario Genomics Institute (OGI-196); EU/EFPIA/OICR/McGill/KTH/Diamond Innovative Medicines Initiative 2 Joint Undertaking (EUbOPEN grant no: 875510); Janssen, Merck KGaA, Merck Sharp and Dohme, Novartis Pharma AG; Pfizer, São Paulo Research Foundation-FAPESP, Takeda and the Wellcome Trust. Additional funding for the SGC-UNC was provided by The Eshelman Institute for Innovation, UNC Lineberger Comprehensive Cancer Center, PharmAlliance, and National Institutes of Health (1R44TR001916-02, 1R01CA218442-01, and 1U24DK116204-01). Support for C.I.W. and D.H.D. also provided by the Foundation for Food and Agriculture Research (FFAR), project A18-2129-S001. Support for I.D. and A.S. provided by DFG, German Reaseach Foundation project 2591307 SFB1177.

Data Availability Statement: Data is contained within the article or supplementary materials.

Acknowledgments: Daniel Ebner and Stephanie B. Hatch would like to acknowledge and thank Vincenzo D'Angiolella, Anderson Ryan, Sarah Blagden, Kristijan Ramadan, Peter McHugh and Tim Humphrey from the University of Oxford, Department of Oncology for their support and hard work in developing and producing the anticancer screening campaigns highlighted in the future direction section of this manuscript. Construction of the KCGS has been highly collaborative and intricate, with contributions large and small from many people. We value and acknowledge the contributions of the many scientists who have made this possible, offered guidance and suggestions, and who indeed will help us continue to improve the set. This list includes Mirra Chung, Jonathan Elkins, Dan Treiber, many scientists in the SGC, and of course the recipients of KCGS and the previous iterations 
of our chemogenomic sets PKIS and PKIS2. These end users have provided valuable feedback that guides our work. Their creative use of the sets provides inspiration to continue down this path so that with ever improving tools the scientific community can link targets to phenotypes and to disease, and through these collaborative efforts identify new targets that will lead to medicines for patients.

Conflicts of Interest: The funders (Eshelman Institute for Innovation, AstraZeneca, Boehringer Ingelheim, Novartis Institute for Biomedical Research, Bayer Pharma, AbbVie, Pfizer Inc., Takeda California, Inc., MSD) provided support in the form of salaries for authors (Eshelman Institute for Innovation-D.H.D., C.I.W., A.D.A., W.J.Z., T.M.W.; AstraZeneca-D.M.A.; Novartis Institute for Biomedical Research-M.F.; Bayer Pharma-U.L.; AbbVie-M.M.; Pfizer Inc.-D.O.; Takeda California, Inc.-K.S.S.; MSD-C.F.), but did not play a role in the study design, data collection and analysis, decision to publish, or preparation of the manuscript. N.S.G. is a founder, science advisory board member (SAB) and equity holder in Gatekeeper, Syros, Petra, C4, Allorion, Jengu, B2S and Soltego (board member). The specific roles of all authors are articulated in the author contributions section.

\section{References}

1. Roskoski, R., Jr. FDA-Approved Small Molecule Protein Kinase Inhibitors. Available online: http://www.brimr.org/PKI/PKIs. htm (accessed on 1 December 2020).

2. Morphy, R. Selectively nonselective kinase inhibition: Striking the right balance. J. Med. Chem. 2010, 53, 1413-1437. [CrossRef] [PubMed]

3. Ferguson, F.M.; Gray, N.S. Kinase inhibitors: The road ahead. Nat. Rev. Drug Discov. 2018, 17, 353-377. [CrossRef] [PubMed]

4. Knapp, S.; Arruda, P.; Blagg, J.; Burley, S.; Drewry, D.H.; Edwards, A.; Fabbro, D.; Gillespie, P.; Gray, N.S.; Kuster, B.; et al. A public-private partnership to unlock the untargeted kinome. Nat. Chem. Biol. 2013, 9, 3-6. [CrossRef] [PubMed]

5. Fedorov, O.; Muller, S.; Knapp, S. The (un)targeted cancer kinome. Nat. Chem. Biol. 2010, 6, 166-169. [CrossRef]

6. Edwards, A.M.; Isserlin, R.; Bader, G.D.; Frye, S.V.; Willson, T.M.; Yu, F.H. Too many roads not taken. Nature 2011, 470, 163-165. [CrossRef]

7. Oprea, T.I.; Bologa, C.G.; Brunak, S.; Campbell, A.; Gan, G.N.; Gaulton, A.; Gomez, S.M.; Guha, R.; Hersey, A.; Holmes, J.; et al. Unexplored therapeutic opportunities in the human genome. Nat. Rev. Drug Discov. 2018, 17, 377. [CrossRef]

8. Klaeger, S.; Heinzlmeir, S.; Wilhelm, M.; Polzer, H.; Vick, B.; Koenig, P.A.; Reinecke, M.; Ruprecht, B.; Petzoldt, S.; Meng, C.; et al. The target landscape of clinical kinase drugs. Science 2017, 358. [CrossRef]

9. Christmann-Franck, S.; van Westen, G.J.; Papadatos, G.; Beltran Escudie, F.; Roberts, A.; Overington, J.P.; Domine, D. Unprecedently Large-Scale Kinase Inhibitor Set Enabling the Accurate Prediction of Compound-Kinase Activities: A Way toward Selective Promiscuity by Design? J. Chem. Inf. Model. 2016, 56, 1654-1675. [CrossRef]

10. Arrowsmith, C.H.; Audia, J.E.; Austin, C.; Baell, J.; Bennett, J.; Blagg, J.; Bountra, C.; Brennan, P.E.; Brown, P.J.; Bunnage, M.E.; et al. The promise and peril of chemical probes. Nat. Chem. Biol. 2015, 11, 536-541. [CrossRef]

11. Jones, L.H.; Bunnage, M.E. Applications of chemogenomic library screening in drug discovery. Nat. Rev. Drug Discov. 2017, 16, 285-296. [CrossRef]

12. Gautam, P.; Jaiswal, A.; Aittokallio, T.; Al-Ali, H.; Wennerberg, K. Phenotypic Screening Combined with Machine Learning for Efficient Identification of Breast Cancer-Selective Therapeutic Targets. Cell Chem. Biol. 2019, 26, 970-979.e974. [CrossRef] [PubMed]

13. Bamborough, P.; Drewry, D.; Harper, G.; Smith, G.K.; Schneider, K. Assessment of chemical coverage of kinome space and its implications for kinase drug discovery. J. Med. Chem. 2008, 51, 7898-7914. [CrossRef] [PubMed]

14. Posy, S.L.; Hermsmeier, M.A.; Vaccaro, W.; Ott, K.H.; Todderud, G.; Lippy, J.S.; Trainor, G.L.; Loughney, D.A.; Johnson, S.R. Trends in Kinase Selectivity: Insights for Target Class-Focused Library Screening. J. Med. Chem. 2011, 54, 54-66. [CrossRef] [PubMed]

15. Elkins, J.M.; Fedele, V.; Szklarz, M.; Abdul Azeez, K.R.; Salah, E.; Mikolajczyk, J.; Romanov, S.; Sepetov, N.; Huang, X.-P.; Roth, B.L.; et al. Comprehensive characterization of the Published Kinase Inhibitor Set. Nat. Biotech. 2016, 34, 95-103. [CrossRef] [PubMed]

16. Drewry, D.H.; Wells, C.I.; Andrews, D.M.; Angell, R.; Al-Ali, H.; Axtman, A.D.; Capuzzi, S.J.; Elkins, J.M.; Ettmayer, P.; Frederiksen, M.; et al. Progress towards a public chemogenomic set for protein kinases and a call for contributions. PLoS ONE 2017, 12, e0181585. [CrossRef]

17. Drewry, D.H.; Wells, C.I.; Zuercher, W.J.; Willson, T.M. A Perspective on Extreme Open Science: Companies Sharing Compounds without Restriction. SLAS Discov. 2019, 24, 505-514. [CrossRef]

18. Moret, N.; Clark, N.A.; Hafner, M.; Wang, Y.; Lounkine, E.; Medvedovic, M.; Wang, J.; Gray, N.; Jenkins, J.; Sorger, P.K. Cheminformatics Tools for Analyzing and Designing Optimized Small-Molecule Collections and Libraries. Cell Chem. Biol. 2019, 26, 765-777.e763. [CrossRef]

19. Edwards, A.; Morgan, M.; Al Chawaf, A.; Andrusiak, K.; Charney, R.; Cynader, Z.; ElDessouki, A.; Lee, Y.; Moeser, A.; Stern, S.; et al. A trust approach for sharing research reagents. Sci. Transl. Med. 2017, 9. [CrossRef]

20. The Structural Genomics Consortium. Available online: www.thesgc.org (accessed on 1 December 2019). 
21. Fabian, M.A.; Biggs, W.H., 3rd; Treiber, D.K.; Atteridge, C.E.; Azimioara, M.D.; Benedetti, M.G.; Carter, T.A.; Ciceri, P.; Edeen, P.T.; Floyd, M.; et al. A small molecule-kinase interaction map for clinical kinase inhibitors. Nat. Biotechnol. 2005, $23,329-336$. [CrossRef]

22. DiscoverX. scanMAX. Available online: https://www.discoverx.com/services/drug-discovery-development-services/kinaseprofiling/kinomescan/scanmax (accessed on 1 December 2019).

23. Manning, G.; Whyte, D.B.; Martinez, R.; Hunter, T.; Sudarsanam, S. The Protein Kinase Complement of the Human Genome. Science 2002, 298, 1912-1934. [CrossRef]

24. Zwick, M.; Kraemer, O.; Carter, A.J. Dataset of the frequency patterns of publications annotated to human protein-coding genes, their protein products and genetic relevance. Data Brief 2019, 25, 104284. [CrossRef] [PubMed]

25. Carter, A.J.; Kraemer, O.; Zwick, M.; Mueller-Fahrnow, A.; Arrowsmith, C.H.; Edwards, A.M. Target 2035: Probing the human proteome. Drug Discov. Today 2019, 24, 2111-2115. [CrossRef] [PubMed]

26. DAYLIGHT Chemical Information Systems. Available online: www.daylight.com/dayhtml/doc/theory/theory.smarts.html (accessed on 1 December 2019).

27. Daina, A.; Michielin, O.; Zoete, V. SwissADME: A free web tool to evaluate pharmacokinetics, drug-likeness and medicinal chemistry friendliness of small molecules. Sci. Rep. 2017, 7, 42717. [CrossRef] [PubMed]

28. Roskoski, R., Jr. Properties of FDA-approved small molecule protein kinase inhibitors. Pharmacol. Res. 2019, 144, 19-50. [CrossRef]

29. Varma, M.V.; Obach, R.S.; Rotter, C.; Miller, H.R.; Chang, G.; Steyn, S.J.; El-Kattan, A.; Troutman, M.D. Physicochemical space for optimum oral bioavailability: Contribution of human intestinal absorption and first-pass elimination. J. Med. Chem. 2010, 53, 1098-1108. [CrossRef]

30. Montenegro, R.C.; Clark, P.G.; Howarth, A.; Wan, X.; Ceroni, A.; Siejka, P.; Nunez-Alonso, G.A.; Monteiro, O.; Rogers, C.; Gamble, V.; et al. BET inhibition as a new strategy for the treatment of gastric cancer. Oncotarget 2016, 7, 43997-44012. [CrossRef]

31. Howarth, A.; Schroder, M.; Montenegro, R.C.; Drewry, D.H.; Sailem, H.; Millar, V.; Muller, S.; Ebner, D.V. HighVia—A Flexible Live-Cell High-Content Screening Pipeline to Assess Cellular Toxicity. SLAS Discov. 2020, 25, 801-811. [CrossRef]

32. Hafner, M.; Niepel, M.; Chung, M.; Sorger, P.K. Growth rate inhibition metrics correct for confounders in measuring sensitivity to cancer drugs. Nat. Methods 2016, 13, 521-527. [CrossRef]

33. Mills, C.; Gerosa, L. Optimized Experimental and Analytical Tools for Reproducible Drug-Response Studies. Available online: http:/ /lincs.hms.harvard.edu/wordpress/wp-content/uploads/2018/06/DoseResponseNanocourse_2018_Final.pdf (accessed on 1 December 2019).

34. Hafner, M.; Niepel, M.; Subramanian, K.; Sorger, P.K. Designing Drug-Response Experiments and Quantifying their Results. Curr Protoc. Chem. Biol. 2017, 9, 96-116. [CrossRef]

35. Stolz, A.; Putyrski, M.; Kutle, I.; Huber, J.; Wang, C.; Major, V.; Sidhu, S.S.; Youle, R.J.; Rogov, V.V.; Dotsch, V.; et al. Fluorescencebased ATG8 sensors monitor localization and function of LC3/GABARAP proteins. EMBO J. 2017, 36, 549-564. [CrossRef]

36. Dikic, I.; Elazar, Z. Mechanism and medical implications of mammalian autophagy. Nat. Rev. Mol. Cell Biol. 2018, 19, 349-364. [CrossRef] [PubMed]

37. Kaizuka, T.; Morishita, H.; Hama, Y.; Tsukamoto, S.; Matsui, T.; Toyota, Y.; Kodama, A.; Ishihara, T.; Mizushima, T.; Mizushima, N. An Autophagic Flux Probe That Releases an Internal Control. Mol. Cell 2016, 64, 835-849. [CrossRef] [PubMed]

38. Ozfiliz-Kilbas, P.; Sarikaya, B.; Obakan-Yerlikaya, P.; Coker-Gurkan, A.; Arisan, E.D.; Temizci, B.; Palavan-Unsal, N. Cyclindependent kinase inhibitors, roscovitine and purvalanol, induce apoptosis and autophagy related to unfolded protein response in HeLa cervical cancer cells. Mol. Biol. Rep. 2018, 45, 815-828. [CrossRef] [PubMed]

39. Zhou, Z.R.; Yang, Z.Z.; Wang, S.J.; Zhang, L.; Luo, J.R.; Feng, Y.; Yu, X.L.; Chen, X.X.; Guo, X.M. The Chk1 inhibitor MK-8776 increases the radiosensitivity of human triple-negative breast cancer by inhibiting autophagy. Acta Pharmacol. Sin. 2017, 38, 513-523. [CrossRef]

40. Liu, Z.; Wang, F.; Zhou, Z.W.; Xia, H.C.; Wang, X.Y.; Yang, Y.X.; He, Z.X.; Sun, T.; Zhou, S.F. Alisertib induces G2/M arrest, apoptosis, and autophagy via PI3K/Akt/mTOR- and p38 MAPK-mediated pathways in human glioblastoma cells. Am. J. Transl. Res. 2017, 9, 845-873. [PubMed]

41. Wang, Y.; Zhang, H. Regulation of Autophagy by mTOR Signaling Pathway. Adv. Exp. Med. Biol. 2019, 1206, 67-83. [CrossRef]

42. Li, Z.Y.; Zhang, X. Kinases Involved in Both Autophagy and Mitosis. Int. J. Mol. Sci. 2017, 18, 1884. [CrossRef]

43. Vasta, J.D.; Corona, C.R.; Wilkinson, J.; Zimprich, C.A.; Hartnett, J.R.; Ingold, M.R.; Zimmerman, K.; Machleidt, T.; Kirkland, T.A.; Huwiler, K.G.; et al. Quantitative, Wide-Spectrum Kinase Profiling in Live Cells for Assessing the Effect of Cellular ATP on Target Engagement. Cell Chem. Biol. 2018, 25, 206-214.e211. [CrossRef]

44. Open Babel: The Open Source Chemistry Toolbox. Available online: http:/ / openbabel.org/ (accessed on 1 December 2019).

45. MATLAB. Available online: https://www.mathworks.com/products/matlab.html (accessed on 1 December 2019).

46. Bhullar, K.S.; Lagaron, N.O.; McGowan, E.M.; Parmar, I.; Jha, A.; Hubbard, B.P.; Rupasinghe, H.P.V. Kinase-Targeted cancer therapies: Progress, challenges and future directions. Mol. Cancer 2018, 17, 48. [CrossRef]

47. Kannaiyan, R.; Mahadevan, D. A comprehensive review of protein kinase inhibitors for cancer therapy. Expert. Rev. Anticancer Ther. 2018, 18, 1249-1270. [CrossRef]

48. Burdova, K.; Yang, H.B.; Faedda, R.; Hume, S.; Chauhan, J.; Ebner, D.; Kessler, B.M.; Vendrell, I.; Drewry, D.H.; Wells, C.I.; et al. E2F1 proteolysis via SCF-cyclin F underlies synthetic lethality between cyclin F loss and Chk1 inhibition. EMBO J. 2019, 38. [CrossRef] [PubMed] 
49. Guo, W.; Vandoorne, T.; Steyaert, J.; Staats, K.A.; Van Den Bosch, L. The multifaceted role of kinases in amyotrophic lateral sclerosis: Genetic, pathological and therapeutic implications. Brain 2020, 143, 1651-1673. [CrossRef] [PubMed]

50. Guttuso, T., Jr.; Andrzejewski, K.L.; Lichter, D.G.; Andersen, J.K. Targeting kinases in Parkinson's disease: A mechanism shared by LRRK2, neurotrophins, exenatide, urate, nilotinib and lithium. J. Neurol. Sci. 2019, 402, 121-130. [CrossRef] [PubMed]

51. Singh, R.M.; Cummings, E.; Pantos, C.; Singh, J. Protein kinase C and cardiac dysfunction: A review. Heart Fail. Rev. 2017, 22, 843-859. [CrossRef] 